\title{
The consultation: expectations versus what can be offered in hair transplant surgery
}

\author{
Antonio S. Ruston, MD Sao Paulo, Brazil
}

One of the most common topics in our daily routine is how to deal with the different degrees of expectation from each patient as well as measure and detect difficult and demanding patients in order to minimize possible frustrations and future dissatisfaction.

We know that each patient will have a different result in terms of density, which depends on the characteristics of the donor area (density, hair type, percentage of follicular units of 1,2, and 3 hairs, and elasticity) as well as in the recipient area to be treated (size and location).

We also know that each patient is unique with varying degrees of demand and different expectations.

In our daily practice we have had difficulty in dealing with these expectations more in terms of density than the naturalness of the result.

Knowing that expectation is subjective:

1. How can we guarantee that the patient will be satisfied with the results in terms of density?

2. How can we quantify this?

3. What can we promise and how far can we get in achieving that?

First, we must educate our patients and fully explain the differences of hair density in the donor area, the types of follicular units, the progression of their baldness, and the balance between donor and recipient areas. When possible, we should use layman vocabulary or graphic animation.

Because it is easy to understand, we have been using 3D animations to explain these issues. In one animation, we show that the lower the temporal recession, the lower the density will be and, contrarily, the higher the temporal recession, the greater the density (Figure 1). We have been using this animation to convince patients to accept a higher temporal recession design, because a picture is worth a thousand words.

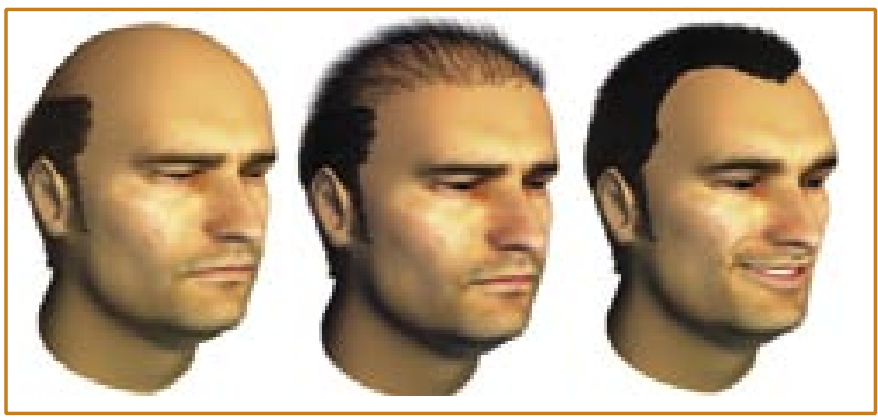

Figure 1. Temporal recession and density

We use another 3D animation to illustrate the effect of the donor area characteristics on the final density and coverage (Figure 2). One forest contains a greater number of trees that are thick, with three branches, and with less distance between the trees. The other contains trees that are thin, with only one or two branches, and with a greater distance among them.

Next, we perform a microscopic analysis of the scalp of our patients showing the different follicular units and giving a score for their donor area in terms of density.

We attempt to characterize the different densities among donor areas as either low, medium, or high hair density (Figure 3).

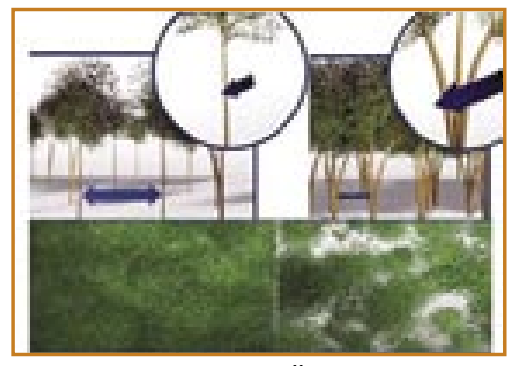

Figure 2. Forest illustration

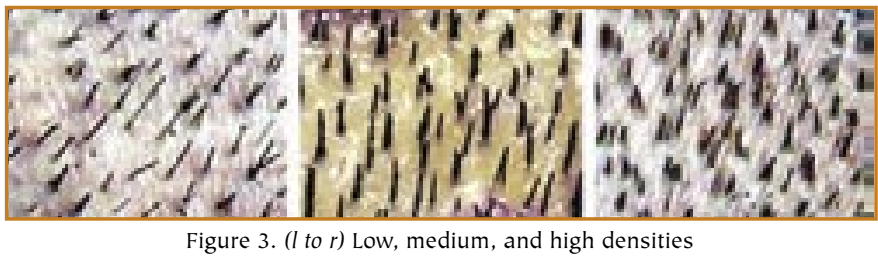

Finally, and most important, we show them their balance between donor and recipient areas.

All of these steps are intended to educate patients and guide them to a more realistic expectation in terms of their result.

After that we try to determine the degree of expectation by showing patients results that we consider poor, average, and exceptionally good in terms of density (Figure 4).

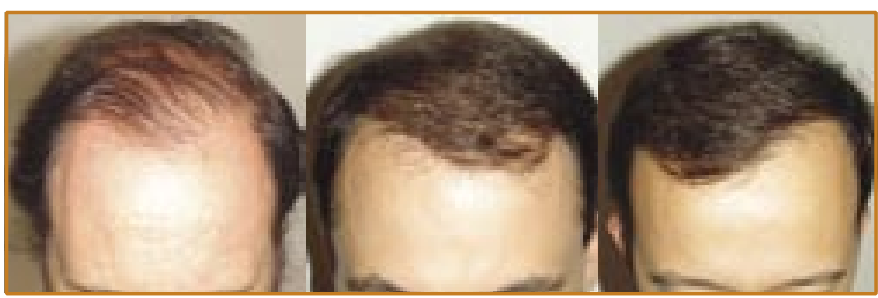

Figure 4. (l to r) Poor, average, and exceptionally good density

During the process of showing each before and after photo we ask our patients if they would be satisfied with that particular result. If they say "no," we present the next result, which is better (denser) than the previous one. This is done until we reach the moment in which the patients say that they would be satisfied with that particular result (or until we reach the last photo, which represents an exceptionally good result for their type of baldness). If after viewing this last photo, and with all of the information provided, the patients say or act in a way that shows they would not be satisfied, this is a warning sign.

In order to facilitate this step, keep your photo database organized by type of baldness, density, hair type, etc.

We have found a number of warning signs that help us measure the difficulty in pleasing our patients:

- Patients who never smile

- Patients who never look directly in your eyes or pay attention to what you are saying

- Patients who look at you suspiciously 
- Patients who do not have any knowledge whatsoever of hair restoration surgery

In our experience, these patients not only make it harder for us in terms of their high expectation levels, but they are also difficult to manage during the procedure. In general this type of patient presents with one or more of the following situations during the procedure:

- They are restless and hard to sedate.

- They ask to go to the bathroom several times.

- They inquire repeatedly about what is being done and if the result will look good.

- They ask about the number of grafts and even still doubt this number.

- They have a very low tolerance for pain and can present more bleeding during the procedure due to anxiety.

In the post-operative period:

- It takes longer for the scabs to fall off.

- The probability of telogen effluvium is greater.

- They complain of more pain than usual.

- They call or email several times to be sure everything that is happening is ok.

These patients will probably have a greater chance of dissatisfaction. Therefore, we recommend that you keep their photos updated to have documentation of the progression of their baldness. In addition, we have to make sure that these patients are taking their oral and/or topical medications. We think it is better not to use software simulation of results in the consultation for these particular patients.

The problem patients may have had bad experiences in the past or perhaps, because they have a higher expectation level, they are disbelievers, mistrustful, or insecure. Independent of the reasons, one thing is certain: There is a direct relationship between patients with high expectations and these types of events.

Even with the most informed patients, they will always "edit" what they want, independent of what the doctor says. Therefore, we must be honest, and never promise beyond that which we can offer.

Remember the $20 / 80$ principle: around $20 \%$ of our patients consume $80 \%$ or more of our energy and our time. So it is fitting for each one of us to define if we wish to be quantitative, qualitative, or selective. However, the most important thing to remember is that once you have accepted this kind of patient, you will have to deal with all these issues. Therefore, you have to embrace the cause. Be patient and treat these patients with the time and attention they require.

In conclusion:

1. Keep the expectations low and be realistic mainly in terms of the coverage that you can achieve because we know that if our patients go into surgery with a lower expectation, the chances are higher that they will be satisfied with the results.

2. We also know that the best results do not always correspond to the most satisfied patients. $\checkmark$

\section{The most advanced hair laser} is now the most affordable.

\section{Advanced \\ Features}

STraturoRers

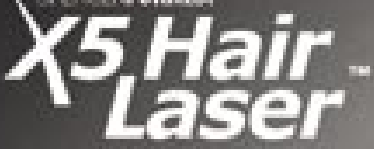

The new $X 5$ delivers low level laser therapy to the scalp at the procesin wavelungth that's deliverad prevan resulta. The $X 5$ is also campletaly cordless. It's designed for grnater confert in artar ts inpone patiant

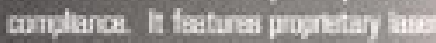
Iyjt phes that bypess nttonening far to dilver bastimitating laner loght with

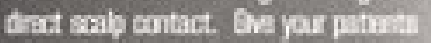
ther best hase uptasn it the trat prise

The Spencer forrest $x 5$ Kailtaser retalls for $\$ 299.00$

Speclal Physiclan prices 5159.95

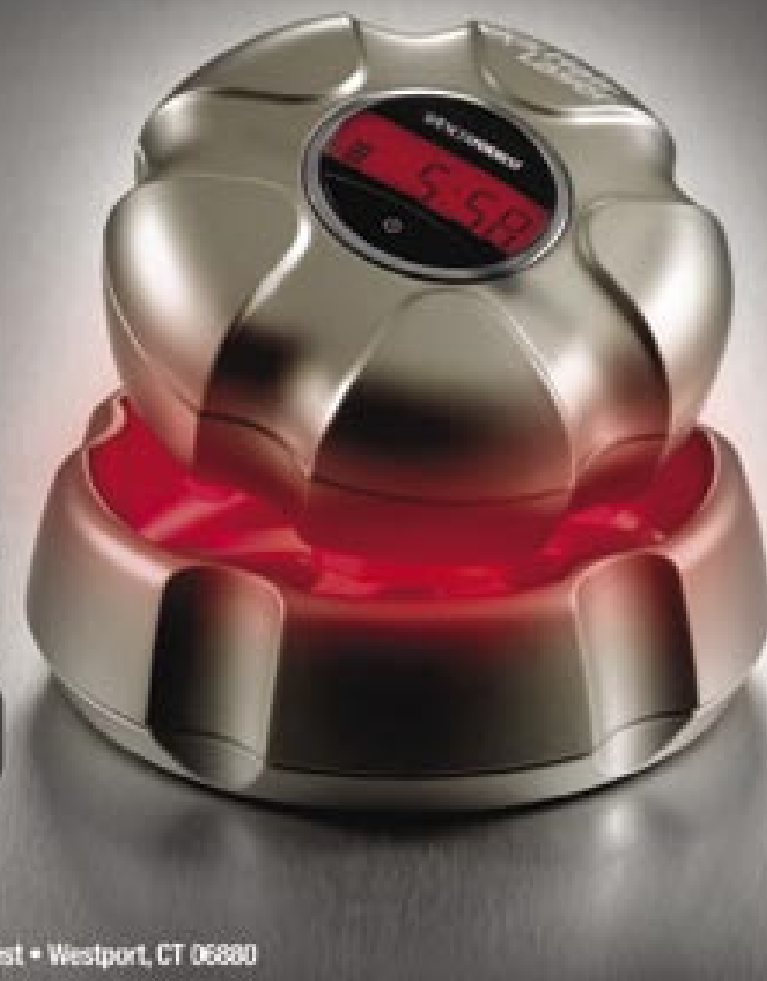

Qcaser.com

1-200-221-7171 fer us and Canada.

Overseas calt $203-221-7171$.

Spencer Forrest Ine * 64 Pust Raid West * Westport, CT 08800

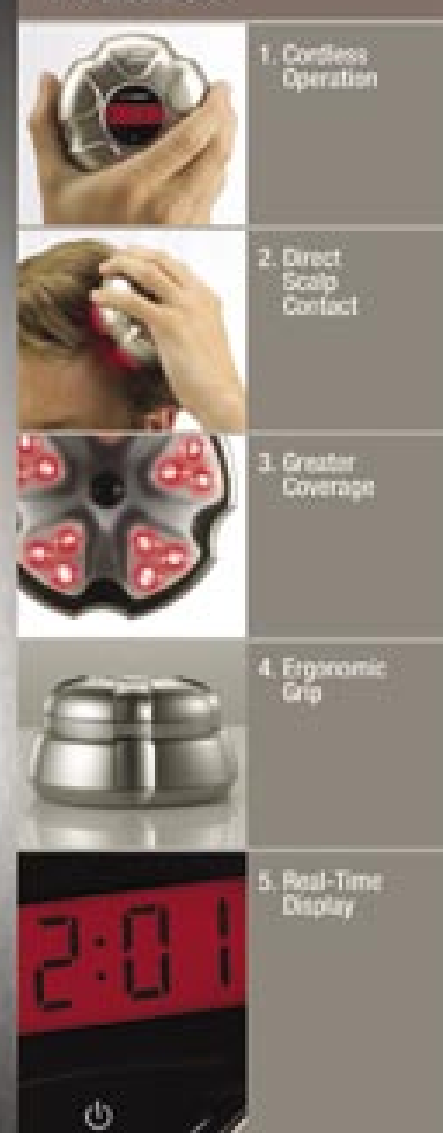

